\title{
Unusual occurrence of cervical myelopathy in a case of Stickler's syndrome
}

\author{
Stéphane Noël, Danielle Balériaux, Nicole Telerman-Toppet
}

\begin{abstract}
We report the occurrence of progressive Brown-Séquard syndrome as the presenting clinical feature of cervical spondylosis in a young patient with Stickler's syndrome.
\end{abstract}

In 1965, Stickler et al ${ }^{1}$ reported the association of progressive myopia and premature degenerative joint changes in a five generation family. This progressive arthro-ophthalmopathy is an autosomal dominant syndrome with great variability of clinical expression. Recently, it has been suggested that a structural abnormality of type II collagen could be the basic defect in this connective tissue disorder. We report here the association of a progressive myelopathy of Brown-Séquard type in a young patient with Stickler's syndrome.

\section{Case report}

A 20 year old man, of Peruvian origin, was admitted to hospital because of a complete loss of temperature sensation on the left side of his body up to the nipple. He complained also of discrete weakness of the right lower limb, of numbness of the left lower limb, and of a dull midline back pain in the interscapular region. These symptoms had started six months before admission.

From early childhood he had severe myopia and had worn glasses from the age of 3 years. $\mathrm{He}$ had also worn a hearing aid from this age because of severe bilateral deafness (100 decibel deficit). At the age of 4 , he was operated upon for epiphyseal dysplasia of the knees. At about 12 years, a spontaneous retinal detachment in the right eye led to five consecutive surgical procedures. Five years later, the lens was extracted and the vitreous replaced by silicone; hyphema and left external rectus paralysis were probable surgical complications. Over the past 10 years, he reported two or three transient episodes of quadriparesis or paraesthesiae of the four limbs induced by minor trauma (forced neck extension). The family history was unrevealing because his mother was asymptomatic and his father died when he was 1 year old.

Physical examination was normal. Neurological examination disclosed intact cranial nerves with the exception of a right external rectus palsy, normal muscle strength and tone, and increased reflexes of the triceps, patellar, and ankle on the right side with a Babinski sign and sustained ankle clonus. The biceps and brachioradialis reflexes were symmetrical. The right abdominal reflexes were absent. On the left side, complete loss of pain and temperature sensation was observed up to the T4 level. Positon and vibration sense and appreciation of light touch were normal.

The laboratory studies were all normal including amino acid screening in blood and urine. Ophthalmoscopic examination of the left eye showed posterior detachment of the vitreous. He also had myopia of 12 diopters. Echocardiography did not show any mitral valve prolapse. Radiographic examination of the skeleton showed spondyloepiphyseal dysplasia with widening of the extremities of the long bones (the femora and tibiae had a dumbbell shape). The femoral epiphysis was flat and associated with a broad femoral neck. Moderate subluxation of the hips, coxa valga, and broadening of the metacarpal and metatarsal heads were also observed, as well as kyphoscoliosis and flattening and irregularity of the vertebral bodies, particularly anterior wedging of the fifth thoracic vertebral body. Cervical spine radiographs showed a congenitally narrow spinal canal and a spina bifida of the atlas. Myelography showed an interruption of the contrast column at the level of C4 to C5. A postmyelogram CT scan showed that the sagittal diameter of the spinal canal was decreased at level $\mathrm{C} 3$ to $\mathrm{C} 7$, with maximum narrowing at the $\mathrm{C} 4$ to $\mathrm{C} 5$ disc where the spinal cord was moderately flattened and displaced posteriorly. Magnetic resonance imaging (MRI) confirmed the cervical canal stenosis and showed the maximum narrowing by osteophyte formation and an area of increased signal intensity in the cervical cord at the level C4 to C5. MRI of the thoracic spine and of the brain were normal. Somatosensory evoked potentials were normal. Brainstem auditory evoked responses, tested with a click stimulus of $100 \mathrm{db}$, showed the absence of all five waves.

\section{Discussion}

Stickler's syndrome is an autosomal dominant hereditary progressive arthro-ophthalmopathy. The incidence is unknown, but the condition is not rare, possibly more common than Marfan syndrome whose incidence is from 4 to 6 per 100000 . A candidate gene for Stickler's syndrome has been proposed in at least some families. The gene, COL2A1, on chromosome 12, encodes type II collagen, a fundamental protein for the structural integrity of many connective tissues affected in Stickler's syndrome. ${ }^{2}$ The disease involves the eyes, the joints, and the orofacial region. The clinical signs related to the eyes are severe myopia, probably congenital, chorioretinal degeneration progressing to spontaneous retinal 
detachment, vitreal degeneration, and cataract. The symptoms of arthropathy are variable and age dependent, like premature osteoarthritis in adulthood. Radiographic findings include epiphyseal dysplasia, widening of the joint spaces, irregularity of the vertebral end plates, and flattening of the vertebral bodies with a tendency to anterior wedging. ${ }^{3}$ Occasional features are thoracic kyphoscoliosis, coxa valga, pes planus, and arachnodactyly with a Marfanoid habitus. ${ }^{45}$ However, height is generally normal. Our patient had short stature $(23 \%$ of patients with Stickler's syndrome are below the 3rd centile for height). ${ }^{4}$ Spina bifida occulta of the atlas, as in our case, is infrequent and not usually mentioned in the occasional features of Stickler's syndrome. Orofacial features are mainly observed in children because many of these signs improve with growth (Pierre-Robin sequence, midfacial hypoplasia). As in our case, the face can appear normal in adulthood. Sensorineural deafness and mitral valve prolapse $^{6}$ have also been reported.

The partial Brown-Séquard syndrome with a sensory level at T4 did not seem initially to reflect a thoracic myelopathy. Radiographic investigations showed a thoracic kyphoscoliosis and irregularity and flattening of the thoracic vertebral bodies with anterior wedging of T5; however, these vertebral changes did not compress the spinal cord. Eventually, they could disturb the blood supply of the vulnerable upper thoracic segments by compression of a radicular artery, but the intervertebral foramina were not narrowed and the patient did not suffer from a thoracic radiculopathy. However, an MRI scan showed cord compression by spondylotic tissues at the $\mathrm{C} 4$ to $\mathrm{C} 5$ level in a congenitally narrow cervical canal. Cervical spondylosis, although unusual in young people with Stickler's syndrome, is not unknown. We consider that the approximation of the adjacent vertebral bodies, owing to an anomaly of the nucleus pulposus or to pathological softness of the vertebral plates, could trigger off a reactive process that produces osteophytes. We could see, on T2 weighted spin echo images, a high signal intensity lesion within the spinal cord at the C4 to C5 level where the narrowing of the cervical canal was maximal (figure). The pathophysiological basis of such an abnormality, found sporadically in cervical spondylosis, is presumed to be myelomalacia or cord gliosis secondary to chronic and gradually increasing compression of the spinal cord. ${ }^{7}$ The most important pathogenetic mechanism for cervical myelopathy in our case is the compression resulting from a constitutionally narrow canal and acquired spondylotic changes. Dynamic components or repeated cord trauma, such as hyperextension injury, may also have caused cord damage. In this way, the spinal cord micro-circulation could be compromised and ischaemia could be responsible for several symptoms in cervical spondylosis. ${ }^{8}$ The posterolateral white columns, as in our partial Brown-Séquard syndrome, first suffered from minor compression. However, the anatomical localisation of the

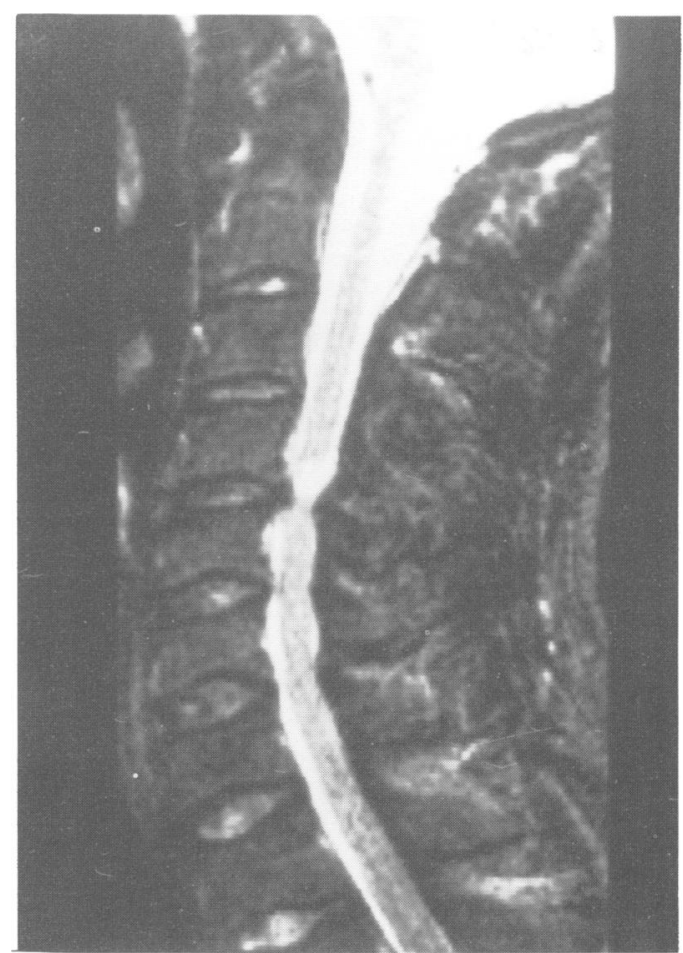

T2 weighted MRI (SE, TR 2500, TE 100) of the cervical spine showing cervical canal stenosis with maximum narrowing and hyperintense area in the cervical cord at the level C4 to C5.

spinal cord lesion by MRI scan, which showed high signal intensity at the level C4 to C5 where the spinal cord was constricted by encroachment of an osteophyte formation, does not square perfectly with the neurological findings, particularly the T4 sensory level. Thus, we must consider that the narrowing of the spinal canal from $\mathrm{C} 3$ to $\mathrm{C} 7$ caused cord damage principally at the $\mathrm{C} 7$ level by the different mechanisms mentioned above, or that the level of sensory loss will ascend as the lesion progresses. This pathology, evolving from the periphery to the centre of the cord, first affects the outermost fibres carrying pain and temperature sensation from the lower extremities.

Harkey et $a l^{9}$ reported the case of a young woman with Stickler's syndrome who developed a flaccid paraparesis and a T6 sensory level in the absence of trauma. This neurological deficit was attributed to a mid-level thoracic disc herniation. The radiographic studies also showed the characteristic degenerative vertebral changes of the disease and a thoracic spinal canal stenosis. A structural defect in type II collagen could probably alter the mechanical properties of the intervertebral disc and cause such herniation in a process of premature disc degeneration. The narrowness of the cervical or thoracic spinal canal may further contribute to the problem and seems to be an important predisposing factor in producing such a cord injury. MRI permits detection and localisation of the early pathological changes of the spinal cord in compression myelopathy. Since the vertebral changes of Stickler's syndrome are most often 
asymptomatic and usually present in the thoracic region, the new finding in our case is the occurrence of a myelopathy secondary to cervical spine abnormalities, without evidence of trauma.

1 Stickler GB, Belau PG, Farrell FJ, et al. Hereditary progressive arthro-ophthalmopathy. Mayo Clin Proc 1965;99:394 9.

2 Francomano CA, Maumenee I, Liberfarb R, Pyeritz RE Cosegregation of Stickler syndrome and type II collagen gene alleles. Cytogenet Cell Genet 1987;46:615A

3 Stickler FB, Pugh DG. Hereditary progressive arthro-ophthalmopathy. Mayo Clin Proc 1967;24:387-92.
4 Temple IK. Stickler's syndrome. $\mathcal{f}$ Med Genet 1989;26:11926.

5 Liberfarb RM, Hirose T, Holmes LB. The Wagner-Stickler syndrome: astudy of 22 families. J Pediatr 1981;99:394-9. Liberfarb RM, Goldblatt A. Prevalence of mitral valve prolapse in the Stickler syndrome. Am $\mathrm{f}$ Med Genet 1986;24:387-92.

7 Takahashi M, Sakamoto Y, Miyawaki M, Bussaka H. Increased MR signal intensity secondary to chronic cervical cord compression. Neuroradiology 1987;29:550-6.

8 Griffiths IR. Some aspects of pathology and pathogenesis of the myelopathy caused by disc protrusions in the dog. $f$ Neurol Neurosurg Psychiatry 1972;35:403-13.

9 Harkey HL, Cullom ET, Parent AD. Thoracic disc herniation and paraplegia in Stickler's syndrome. Neurosurgery tion and paraple 